\title{
A Miniaturized Non-Radioactive Electron Emitter Including High Vacuum Pressure Monitoring
}

\author{
P. Cochems, M. Bellmann, M. Runge, S. Zimmermann \\ Inst. of Electrical Engineering and Measurement Technology \\ Department of Sensors and Measurement Technology, Leibniz University Hannover \\ Hannover, Germany \\ cochems@geml.uni-hannover.de
}

\begin{abstract}
:
We present a miniaturized non-radioactive electron source based on thermo emission with integrated high vacuum pressure monitoring. This electron source generates free electrons at atmospheric pressure to be an alternative to radioactive beta minus sources. Most gas sensor systems based on atmospheric pressure chemical ionization (ACPI), such as ion mobility spectrometers, use radioactive beta minus sources to provide free electrons with high kinetic energy to initiate a chemical gas phase ionization of the analytes to be detected. Instead of the typically used radioactive beta minus sources to provide high energetic electrons, we generate free electrons in vacuum by thermionic emission from an electrically heated tungsten filament. The electrons emitted from the filament are then accelerated towards an electron transparent, but gas tight $\mathrm{Si}_{3} \mathrm{Ni}_{4}$-membrane, through which the electrons are transferred from vacuum to atmospheric pressure. An insufficient vacuum in the miniaturized nonradioactive electron source may cause electrical breakdown, which not only produces electromagnetic interference but can also damage the $\mathrm{Si}_{3} \mathrm{~N}_{4}$-membrane by decreasing the kinetic energy of the electrons leading to charging effects and extensive energy deposition in the membrane. Therefore, we present a concept to self-monitor the vacuum in the non-radioactive electron source by simply switching the electronics from electron emission to pressure monitoring mode.
\end{abstract}

Key words: Vacuum pressure monitoring, non-radioactive ionization source, ion mobility spectrometry, atmospheric pressure chemical ionization (APCI), trace gas detection

\section{Introduction}

In ion mobility spectrometry (IMS) different gases are detected and identified by ionizing these gases and investigating the motion of their ions through a drift gas, e.g. purified air or nitrogen, under the influence of an electric drift field [1]. Generally speaking, IMS is a technique for fast trace gas detection within seconds and detection limits in the very low ppb- and even ppt-range. Furthermore, IMS scores with its minor instrumental effort compared to its very good analytical performance. In most IMS radioactive beta minus sources, e.g. ${ }^{3} \mathrm{H}$ or ${ }^{63} \mathrm{Ni}$, are used to provide high energetic electrons. These electrons initiate an atmospheric pressure chemical ionization (APCI), which is the key to ultra-low detection limits. A detailed description of APCl can be found in [1] and [2]

One advantage of radioactive beta minus decay sources is that no external power supply or any other instrumentation is needed to emit high energetic electrons. This leads to a minimum of weight, size and power consumption and requires no maintenance. However, adjustable ionization parameters like kinetic electron energy, ionization time, electron current and density open new possibilities, e.g. to investigate the reaction kinetics of ion formation and ion-ion recombination for the development of new ion separation techniques [3, 4], that are impossible with radioactive sources. Moreover, in many applications the use of radioactive sources is simply not permitted or difficult due to legislative concerns.

Therefore, we develop a non-radioactive electron source. A detailed description can be found in [5]. To provide free electrons at atmospheric pressure having a kinetic energy in the range of the kinetic energy of electrons emitted from ${ }^{63} \mathrm{Ni}$ or ${ }^{3} \mathrm{H}$ sources, the electrons have to be accelerated to the desired kinetic energy in an electric field inside a vacuum chamber to prevent electric breakdown. ${ }^{3} \mathrm{H}$ emits electrons with a maximum kinetic energy of $18.6 \mathrm{keV}$ and an average kinetic energy of $5.7 \mathrm{keV}$ [5]; ${ }^{63} \mathrm{Ni}$ emits faster electrons than ${ }^{3} \mathrm{H}$ with a maximum kinetic energy of $67 \mathrm{keV}$ and an average kinetic energy of $17 \mathrm{keV}$ [6]. To 
generate free electrons in a vacuum chamber thermionic emission [7] is used. Therefore, a thermionic emitter is heated up to stimulate electrons in the solid state. An applied electric extraction field provides additional energy to the electrons to eventually overcome the work function. Thus, electrons can leave the solid state. For energized free electrons at atmospheric pressure the electrons need to be transferred from the vacuum to atmospheric pressure at the end of the acceleration region. A gas tight, but electron transparent $\mathrm{Si}_{3} \mathrm{~N}_{4}$ membrane is used in our setup. With this electron source coupled to our IMS as shown in Fig. 1 we can easily reach an electron current at atmospheric pressure of about $50 \mathrm{pA}$. This electron emission current corresponds to the activity of a $300 \mathrm{MBq}{ }^{3} \mathrm{H}$ source, which is used in the cited references as an ionization source for IMS.

To avoid electrical breakdown and electron collisions with residual gas, a good vacuum in the electron source is needed. Collisions of the electrons with residual gas decrease the kinetic energy of the electrons. The $\mathrm{Si}_{3} \mathrm{~N}_{4}$-membrane may be destroyed by electrons with an unfavorable low kinetic energy [8]. Furthermore, electrical breakdown cause high frequency electromagnetic interference and may cause interference or even destruction of the electronics. To protect the $\mathrm{Si}_{3} \mathrm{~N}_{4}$-membrane and the surrounding circuits the pressure inside the electron source must be monitored.

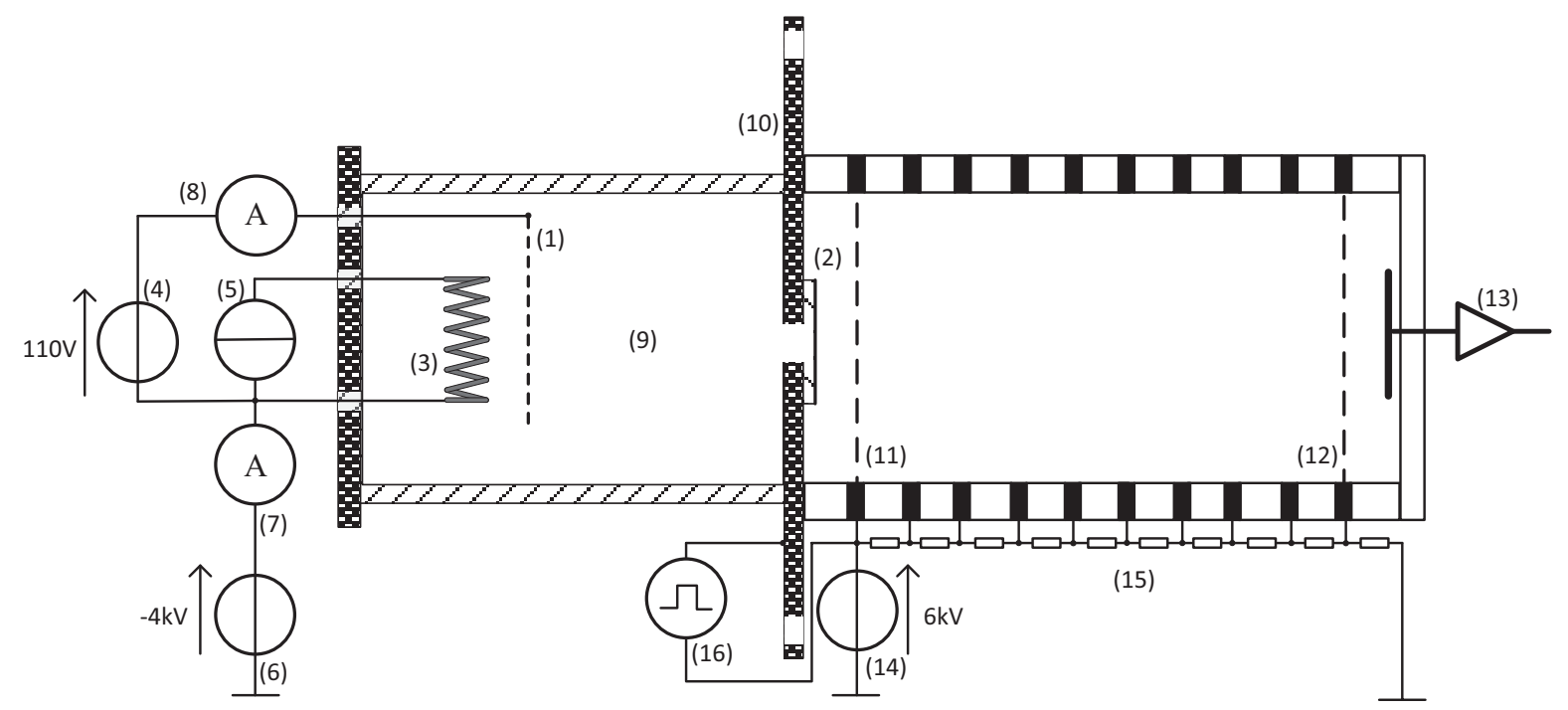

Fig. 1: Schematic diagram of the miniaturized non-radioactive electron source coupled to a time-of-flight IMS including extraction grid (1), $\mathrm{Si}_{3} \mathrm{~N}_{4}$-membrane (2), tungsten filament (3), grid voltage source (4), filament heating source (5), acceleration voltage source (6), emission current measurement (7), grid current measurement (8), vacuum chamber (9), electron source cover (10), injection grid (11), aperture grid (12), current amplifier (13), drift voltage source (14), voltage divider (15), injection pulse source (16).

Because a good vacuum is so important: The IMS measurement system should be able to automatically check the vacuum, or measure the pressure inside the vacuum chamber respectively, before ramping up the high voltage sources. To achieve this requirement the pressure measurement must be integrated into the electron source and its environment.

\section{Concept}

Due to the non-radioactive electron emitter construction it is appropriate to also use the thermionic electron emitter to ionize the residual gas in the vacuum chamber like most state of the art vacuum gauges [9]. In this gauges the ratio between the electron emission current $i$ from the thermionic emitter to the ion current $i^{+}$ on a detector is measured and multiplied by a geometrical constant $C$ to determine the pressure $p$ as shown in Eq. 1.

$$
p=\frac{i^{+}}{i^{-}} C
$$

To measure and control the electron emission current in our set up during pressure monitoring mode the grid current is measured and the filament heating current is controlled by a $\mathrm{PI}$-controller to reach the desired electron emission current. For pressure monitoring the grid has the highest potential inside the vacuum chamber, so that all of the emitted electrons are collected at the grid (shown in Fig. 2). 


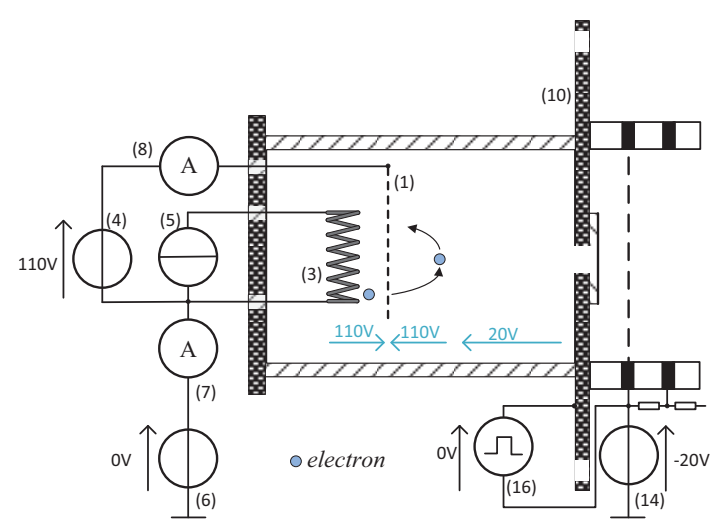

Fig. 2: Miniaturized non-radioactive electron emitter in pressure monitoring configuration. After thermionic emission all electrons eventually hit the grid. Thus, the electron emission current can measured at the grid.

Thus, the emission current measurement (7), which is used in electron source mode to measure the electron emission current, now measures the residual gas ion current. This ion current together with the electron emission current, which is measured in pressure monitoring mode with the grid current measurement (8) enables us to measure the pressure inside the vacuum chamber.

One known issue of the pressure measurement as shown in Fig. 2 is the photo emission current from the cover (10) caused by bremsstrahlung from the electrons hitting the grid. This photo current cannot be distinguished from the ion current caused by residual gas ionization. Thus, this pressure measurement method is limited to pressures of about $10^{-5} \mathrm{mbar}$ and above in our setup. To improve the pressure measurement we simply integrated one additional electrode into the vacuum chamber, which can be switched between the grid potential and a potential of $20 \mathrm{~V}$ below the filament. This method is described in detail in [10], where the additional electrode is called ion modulator electrode (Fig. 3).

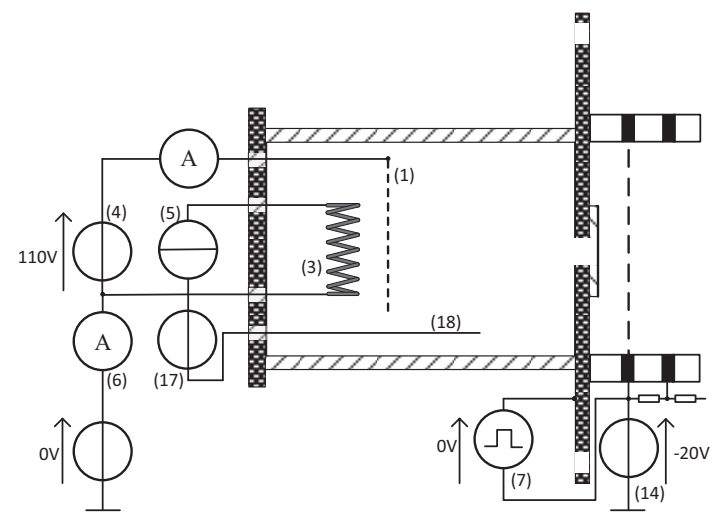

Fig. 3: Miniaturized non-radioactive electron emitter with an integrated ion modulator electrode (18) and a modulator voltage source (17) to improve the pressure measurement.

This electrode collects a constant fraction of the total ion current when it has an electric potential below the filament, while it collects no ions when having grid potential. The measured ion current (7) with the ion modulator electrode at grid voltage can be described as follows

$$
I_{g}=i^{+}+i^{r}
$$

where $I_{g}$ is the measured current (7) when the ion modulator electrode is at grid voltage, $i^{+}$is the positive ion current caused by residual gas ionization and $i^{r}$ is the residual current caused by photo emission. Correspondingly, when the ion modulator electrode is switched to a potential $20 \mathrm{~V}$ below the filament the measured current $I_{-20}$ can be described as

$$
I_{-20}=m \cdot i^{+}+i^{r}
$$

The factor $m$ is a pressure independent modulation factor. The modulation factor can be found at higher pressures, where $i^{r}$ is much smaller than $i^{+}$. After measuring $m$ the positive ion current can be calculated by Eq. 4 .

$$
i^{+}=\frac{I_{g}-I_{-20}}{1-m}
$$

As shown in Eq. 4, only the difference between the current measured with the ion modulator electrode at grid potential and $20 \mathrm{~V}$ below the filament is needed. Thus, any current measurement offsets are eliminated and do not need to be considered.

\section{Experimental Setup}

In order to demonstrate the feasibility of this pressure self-monitoring concept we evacuated the miniaturized non-radioactive electron source by a HiCube 80 vacuum pump from Pfeiffer Vacuum. To adjust the pressure from $10^{-6} \mathrm{mbar}$ to $10^{-4} \mathrm{mbar}$ a needle valve from Pfeiffer Vacuum is used. The pressure reference gauge is a Pfeiffer Vacuum PKR-251. For operating and controlling the nonradioactive electron source in electron emission and pressure monitoring mode we used our own electronics.

\section{Results and Discussion}

With the experimental setup described in Fig. 2 vacuum pressures down to $10^{-5}$ mbar could be measured. Fig. 4 shows the measurement results without using the ion modulator electrode. The increasing dashed curve fits the ion current very well down to $10^{-5}$ mbar. At $10^{-5}$ mbar the photo emission current dominates the positive ion current and no pressure measurement below $10^{-5}$ mbar is possible in our setup. 


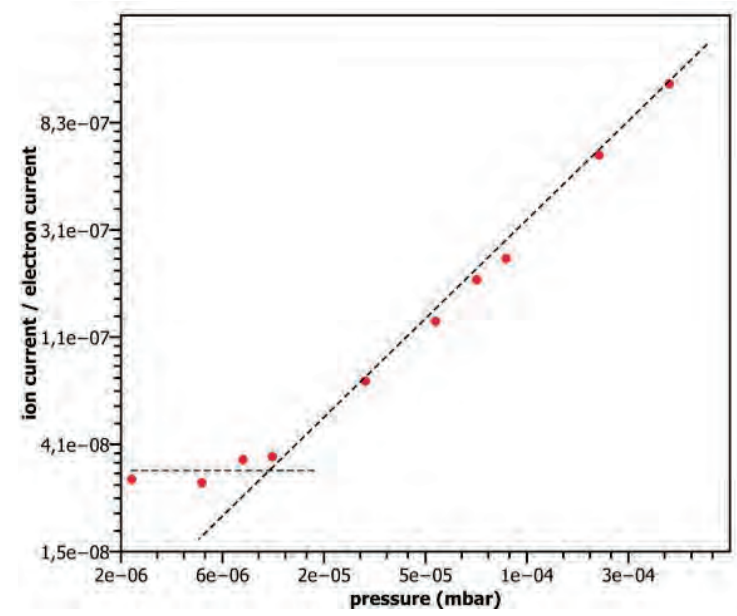

Fig. 4: Measured ion current per electron current vs. pressure (no ion modulator electrode).

Fig. 5 shows the measurement results when using the ion modulator electrode.

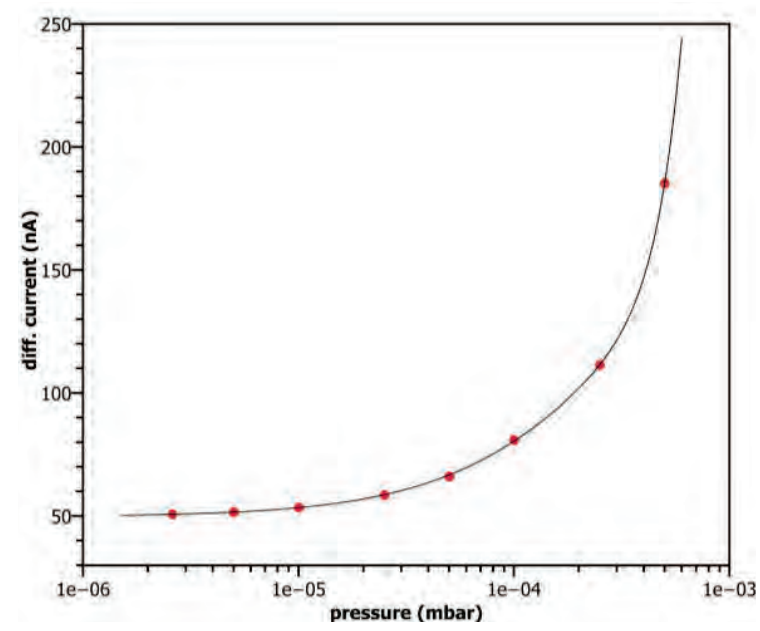

Fig. 5: Measured difference current vs. pressure with ion modulator electrode.

The pressure measurement could be improved by an order of magnitude. Furthermore, the reproducibility could be increased which leads to much better pressure resolution.

\section{Conclusion}

In this paper we present a pressure selfmonitoring concept to monitor the pressure inside the vacuum part of our miniaturized nonradioactive electron source. The electron generation is based on thermionic emission from a tungsten filament, which is also used in pressure monitoring mode to generate free electrons to ionize residual gas molecules in the vacuum chamber. It has been demonstrated that this pressure self-monitoring concept allows to measure pressures from about $10^{-6} \mathrm{mbar}$ up to $10^{-3} \mathrm{mbar}$. Furthermore, our concept allows automatically monitoring the vacuum before ramping up the high voltage source for electron acceleration by simply switching the electronics from electron emission to pressure monitoring mode. Thus, it is possible to prevent damages to the nonradioactive electron source and to other electronics by electromagnetic interference caused by an electric breakdown.

\section{References}

[1] G. Eiceman and Z. Karpas, Ion mobility spectrometry, CRC Press, 2005.

[2] H. Borsdorf und G. Eiceman, „Ion Mobility Spectrometry: Principles and Applications," Applied Spectroscopy Review, p. 41:323 375, 2006.

[3] A. Heptner, P. Cochems, J. Langejuergen, F. Gunzer and S. Zimmermann, "Investigation of ion-ion-recombination at atmospheric pressure with a pulsed electron gun," Analyst, p. 137:5105 5112, 2012.

[4] P. Cochems, F. Gunzer, J. Langejuergen, A. Heptner and S. Zimmermann, "Selective ion suppression as a pre-separation method in ion mobility spectrometry using a pulsed electron gun," Int. J. Ion Mobil. Spec., p. 15:31 39, 2012.

[5] P. Cochems, M. Runge und S. Zimmermann, „A miniaturized nonradioactive electron emitter for atmospheric pressure chemical ionization," Transducers 2013, 2013.

[6] F. T. Porter, „Beta Decay Energy of Tritium," Physical Review, p. 450:453, 1959.

[7] D. W. Hetherington, R. L. Graham, M. A. Lone, J. S. Geiger und G. E. Lee-Whiting, „Upper Limits on the mixing of heavy neutrinos in the beta decay of $63 \mathrm{Ni}$," Physical Review C, p. 1504:1513, 1987.

[8] W. B. Nottingham and G. Eastman, "Thermionic Emission from Tungsten and Thoriated Tungsten Filaments," Physical Review, p. 78:97, 1936.

[9] A. Morozov, T. Heindl, C. Skrobol, J. Wieser, R. Kruecken and A. Ulrich, "Transmission of $10 \mathrm{keV}$ electron beams through thin ceramic foils: Measurements and Monte Carlo simulations of electron energy distribution functions," Eur. Phys $J$ D, p. $48: 383$ 388, 2008.

[10] R. T. Bayard und D. Alpert, „Extension of the low pressure range of the ionization gauge," Rev. Sci. Instrum., pp. nr. 21, p. 571, 1950.

[11] P. A. Redhead, „Modulated BayardAlpert Gauge," Rev. Sci. Instrum. 31, p. doi: 10.1063/1.1716973, 1960. 